\title{
Survey for $\beta$-lactamase among bacterial isolates from Guangzhou, China hospitals between 2005-2006
}

\author{
Weijiao Liao, Jiehua Jiang, Yunjian Xu, Jianyun Yi, Tao Chen, Xiuxin Su, Sizheng Pan, Xiaoping Wei \\ and Yiquan Li
}

The $\beta$-lactamase genes, which confer multi-drug resistance, are spreading among clinical bacterial isolates. As part of a routine surveillance program, we collected 302 bacilli isolates between June 2005 and October 2006 from four hospitals in Guangzhou, China. The isolates were screened for multidrug resistance and for the presence of $\beta$-lactamases. In all, 80 isolates were identified as multidrug-resistant with the K-B method. These isolates were phenotypically screened for $\beta$-lactamase activity by disk diffusion prescreening, diffusion confirmation, the cefoxitin three-dimensional test and the metallo- $\beta$-lactamase (MBL) synergy test. Bacteria were genotypically screened for $\beta$-lactamase genes by PCR and DNA sequencing. Among the 80 strains, drug resistance was lowest to amikacin $(18.75 \%)$ and highest to ampicillin $(97.50 \%), 26.49 \%$ had a $\beta$-lactamase phenotype, $16.56 \%$ had the extended-spectrum $\beta$-lactamase (ESBL) phenotype, $24.83 \%$ had a $\beta$-lactamase genotype, 51 carried integrons, 30 carried class I integrons and $18.75 \%$ had the ISEcp1B insertion sequence. Sequencing also detected a new CTX-M ESBL gene subtype, which had an ISEcp1B element upstream of bla composite transposon. Multidrug resistance and $\beta$-lactamases continue to be prevalent in Guangzhou. Our results suggest that resistance genes are evolving and being horizontally transmitted between species.

The Journal of Antibiotics (2010) 63, 225-229; doi:10.1038/ja.2010.23; published online 26 March 2010

Keywords: $\beta$-lactamases; genotype; integron; insertion sequence; transposon

\section{INTRODUCTION}

In the past 20 years, a large number of cephalosporins have been developed. In response, a large number of multidrug-resistant strains of bacteria have emerged. Cephalosporins are broad-spectrum antibacterials, and widespread use of these antibiotics is believed to have created selective pressure on bacteria to gain resistance to cephalosporins. Among the Gram-negative bacilli, the production of the $\beta$-lactamase enzymes is one of the main mechanisms of resistance to cephalosporins. The $\beta$-lactamases are being transmitted from species to species among the bacteria, and as 'selfish genes', the $\beta$-lactamases themselves are undergoing rapid evolution. The $\beta$-lactamases have a broad and increasing spectrum of substrates, are increasing in genetic varieties, and spread easily.

Ambler et al. ${ }^{1}$ classified the $\beta$-lactamases into four types, A, B, C and D, according to amino acid sequence. In 1995, expanding on Ambler's molecular classification method, Bush et al. ${ }^{2}$ further classified the $\beta$-lactamases into four categories based on substrate specificity as well as the sensitivity to clavulanic acid and EDTA. The extended-spectrum $\beta$-lactamase (ESBL), cephalosporinase AmpC and metallo- $\beta$-lactamase (MBL) are the commonly seen varieties in clinical practice.

$\beta$-lactamases may be transmitted via plasmids and transposons, and the transmission of DNA via conjugation and transposition.
The appearance of type SHV, DHA and VIM $\beta$-lactamases mediated by transposons, insertion sequences and integrons indicates that the drug-resistance genes can transmit horizontally. ${ }^{3-5}$

Because of the urgent clinical effect of the $\beta$-lactamase genes, continuous monitoring of clinical isolates is needed, especially in high-density population centers such as Guangzhou, which can serve as epicenters for the evolution and transmission of new strains. Here we report the results of a survey of multidrug resistance among bacterial clinical isolates from Guangzhou, China.

\section{MATERIALS AND METHODS}

\section{Bacterial strains}

We isolated 302 non-repetitive Gram-negative bacilli from clinical specimens collected by the laboratories of four hospitals in the Guangzhou area (the First Affiliated Hospital of Guangzhou Medical College, the Second Affiliated Hospital of Guangzhou Medical College, the First People's Hospital in the city of Guangzhou, and the Guangzhou Red Cross Hospital) from June 2005 to October 2006. The bacteria were identified to species with the VITEK-2 identification system (BioMerieux, Marcy l'Etoile, France).

Escherichia coli (E. coli) ATCC25922, Klebsiella pneumoniae (K. pneumoniae) ATCC700603 and Pseudomonas aeruginosa (P. aeruginosa) ATCC27853 were purchased from the identification institution of the Ministry of Health (Beijing, China) as quality control strains. The standard AmpC enzyme 
producing strains of Enterobacter cloacae (E. cloacae) 029 M (chromosomemediated de-repressed continuous high-yielding type) and E. coli DH5 22919 (carrying a plasmid producing ACT-1) were gifts from the Third Affiliated Hospital of Sun Yat-Sen University.

\section{Antimicrobial susceptibility}

Isolates were tested for antibiotic resistance with the VITEK-2 GNP drug susceptibility card (BioMerieux) and with the K-B sensitivity test on MuellerHinton plates with commercial antimicrobial susceptibility disks, strictly following the recommendations of the Clinical and Laboratory Standards Institute (CLSI). ${ }^{6}$ Multidrug-resistant strains were defined as resistant to three or more of the following antimicrobial agents: cephalosporins (ceftazidime (CAZ), CAZ with clavulanic acid, cefotaxime, cefotaxime with clavulanic acid, ceftriaxone, cefazolin, cefepime, cefoperazone, cefoperazone with sulbactam and cefoxitin); penicillins (piperacillin with tazobactam, ampicillin, piperacillin, ampicillin with sulbactam and ticarcillin with clavulanic acid); carbapenems (imipenem and aztreonam); aminoglycosides (gentamicin and amikacin); sulfonamides (cotrimoxazole); and quinolones (ciprofloxacin and levofloxacin).

\section{$\beta$-Lactamase phenotypes}

The strains found to be multidrug resistant were further screened by phenotype for the presence of $\beta$-lactamase. The ESBLs phenotype of the strains was examined using the disk diffusion prescreen and confirmation method recommended by CLSI according to the ESBLs experiment guideline. ${ }^{6}$ The screening of the AmpC enzyme and the identification test were conducted using the cefoxitin three-dimensional test, as previously published. ${ }^{7}$ The MBL synergy test was used for initial screening for the identification of the metalloenzyme; strains with a $>16 \mu \mathrm{g} \mathrm{ml}^{-1} \mathrm{CAZ}$ minimum inhibitory concentration (MIC) value were treated as strains suspected to produce MBL.

\section{$\beta$-Lactamase genotypes}

DNA template samples from isolates with $\beta$-lactamase phenotypes were screened for $\beta$-lactamase genotypes. Briefly, overnight cultures of bacteria were suspended in $60 \mu \mathrm{l}$ of TE buffer $\left(10 \mathrm{mmoll}^{-1}\right.$ Tris $\bullet \mathrm{Cl}, 1 \mathrm{mmoll}^{-1} \mathrm{EDTA}, \mathrm{pH}$ 8.0). The suspension was shaken and mixed well, and then heat-lysed in a $97^{\circ} \mathrm{C}$ water bath $(10 \mathrm{~min})$, placed at $-20^{\circ} \mathrm{C}(1 \mathrm{~min})$ and centrifuged at 12000 r.p.m. for 5 min (Optima L-90K ultra-high-speed refrigerated centrifuge; Beckman, Fullerton, CA, USA). The supernatant was collected and kept at $-20^{\circ} \mathrm{C}$ for later use.

The presence of known $\beta$-lactamase genotypes in the isolates was determined by PCR amplification with specific primers (Table 1) followed by DNA sequencing. The PCR was carried out in a total volume of $20 \mu \mathrm{l}$, consisting of $1 \mu \mathrm{l}$ each of forward and reverse primers, $2 \mu \mathrm{l}$ of $10 \times$ PCR buffer, $1 \mu \mathrm{l}$ of $150 \mu \mathrm{moll}^{-1} \mathrm{dNTP}, 1 \mu \mathrm{l}$ of $2.5 \mathrm{mmoll}^{-1} \mathrm{Mg}^{2+}, 1 \mu \mathrm{l}$ of $1 \mathrm{U}$ Taq enzyme, $2 \mu \mathrm{l}$ of DNA template and $11 \mu \mathrm{H}_{2} \mathrm{O}$. The reaction conditions were: 1 cycle of denaturation at $95^{\circ} \mathrm{C}$ for $3 \mathrm{~min}$; followed by 30 cycles of denaturation at $95^{\circ} \mathrm{C}$ for $30 \mathrm{~s}$, annealing at $56^{\circ} \mathrm{C}$ for $35 \mathrm{~s}$, and extension at $72{ }^{\circ} \mathrm{C}$ for $45 \mathrm{~s}$; and a final extension at $72{ }^{\circ} \mathrm{C}$ for $10 \mathrm{~min}$. Shanghai Yingjun Biological (Shanghai, China) synthesized the primers and sequenced the amplification products. Using BLAST, we compared the sequences with GenBank to determine isolate genotypes.

\section{Genetic environment of the $\beta$-lactamases}

In order to define the mechanism of transmission of the $\beta$-lactamases, we screened our $\beta$-lactamase-positive isolates for integrons and insertion sequences carrying the genes. The variable primer and degenerate primers were used to amplify the integron, then a nested PCR reaction followed by sequence determination was used to look for a class I integron carrying the $\beta$-lactamase gene cassette.

Integrons were detected by PCR with a variable primer pair, 5'-GGCATC CAAGCAGCAAG- $3^{\prime}$ and $5^{\prime}$-AAGCAGACTTGACCTGA-3', ${ }^{\prime 1}$ and a degenerate primer pair, $5^{\prime}$-TGCGGGTYAARGATBTKGAT- ${ }^{\prime}$, $5^{\prime}$-CARCACATGCGTRTAR AT- $3^{\prime}{ }^{4}$ PCR conditions were similar to that mentioned above, with annealing temperatures of $56^{\circ} \mathrm{C}$ for the variable primers, and $50^{\circ} \mathrm{C}$ for the degenerate primers. Known insertion sequences were screened for by nested PCR on the integron amplicons with previously published primers (Table 2).
Table 1 Primers sequences used in this study for genotyping strains

\begin{tabular}{|c|c|c|c|}
\hline Target gene & Primer & DNA sequence $\left(5^{\prime} \rightarrow 3^{\prime}\right)$ & $\begin{array}{l}\text { Amplicon } \\
\text { size (bp) }\end{array}$ \\
\hline \multirow[t]{12}{*}{ AmpC } & ACT-1-F & ACCGTTACGCCGCTGATG & 1326 \\
\hline & ACT-1-B & CCACGAGCTGCCAAACCC & \\
\hline & $\mathrm{DHA}-\mathrm{F}^{\mathrm{a}}$ & ATGGCGGTTGCCGTCTC & 967 \\
\hline & DHA-B & TGACTCTTTCGGTATTCGGGAG & \\
\hline & CMY-G1-F & GCTTACAGCCTCTTTCTCCAC & 1082 \\
\hline & CMY-G1-B & CCTCGACACGGACAGGGTTA & \\
\hline & CMY-G2-F & GGTCTGGCCCATGCAGGTGA & 963 \\
\hline & CMY-G2-B & TGACTTTCGGTATTCGGGTAG & \\
\hline & FOX-F & ATCCAGCCGATGCTCAAGG & 941 \\
\hline & FOX-B & GGCACGAACGCCACATAG & \\
\hline & TEM-F & GTGTCGCCCTTATTCCC & 785 \\
\hline & TEM-B & CATAGTTGCCTGACTCCC & \\
\hline \multirow[t]{14}{*}{ ESBLS } & SHV-F & TGTATTATCTCCCTGTTAGC & 843 \\
\hline & SHV-B & TTAGCGTTGCCAGTGCTC & \\
\hline & CTX-M-G1F & AGTGCAACGGATGATGT & 792 \\
\hline & CTX-M-G1B & GGCTGGGTAAAATAGGTC & \\
\hline & CTX-M-G2F & CGACGCTACCCCTGCTAT & 832 \\
\hline & CTX-M-G2B & CAGAAACCGTGGGTTACGA & \\
\hline & CTX-M-G3F & ACGCTGTTGTTAGGAAGTG & 759 \\
\hline & CTX-M-G3B & TTGAGGCTGGGTGAAGT & \\
\hline & PSE-Fb & AATGGCATTCAGCGCTTCCC & 832 \\
\hline & PSE-B & GGGGCTTGATGCTCACTCC & \\
\hline & OXA-F & TCAACTTTCAAGATCGCA & 1042 \\
\hline & OXA-B & GTGTGTTTAGAATGGTGA & \\
\hline & IMP-1-FC & CTACCGCAGCAGCAGAGTCTTTG & 587 \\
\hline & IMP-1-B & GAACAACCAGTTTTGCCTTACC & \\
\hline \multirow[t]{2}{*}{ MBL } & VIM-1-F & AGTGGTGAGTATCCGACAG & 836 \\
\hline & VIM-1-B & ATGAAAGTGCGTGGAGAC & \\
\hline
\end{tabular}

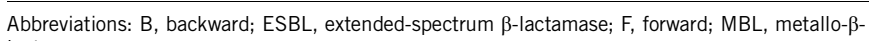
lactamase.

aDHA primers were from Zhang et al..$^{8}$

bPSE primers were from Xiao et al. ${ }^{9}$

cIMP-1 primers were from Han et al 10

Table 2 PCR primers and conditions for amplifying insertion sequences

\begin{tabular}{|c|c|c|c|c|}
\hline Target & Primer & $\begin{array}{l}\text { Oligonucleotide } \\
\text { sequence }\left(5^{\prime} \rightarrow 3^{\prime}\right)\end{array}$ & $\begin{array}{l}\text { Amplicon } \\
\text { length (bp) }\end{array}$ & $\begin{array}{l}\text { Annealing } \\
\text { temp }\left({ }^{\circ} \mathrm{C}\right)\end{array}$ \\
\hline CTX-G-W & $\begin{array}{l}\mathrm{CMF} \\
\mathrm{CMB}\end{array}$ & $\begin{array}{l}\text { CGGAATTCATGGTGACAAAG } \\
\text { AGAGTGCAAC } \\
\text { GCGCTCGAGTTACAGCCCTT } \\
\text { CGGCGAT }\end{array}$ & 876 & 56 \\
\hline ISEcp $1 B$ & $\begin{array}{l}\text { ISEF } \\
\text { ISEB }\end{array}$ & $\begin{array}{l}\text { AAAATGATTGAAAGGTGGT } \\
\text { ACYTTACTGGTRCTGCACAT }\end{array}$ & 546 & 52 \\
\hline IS903 & $\begin{array}{l}903 F \\
903 B \\
903 C\end{array}$ & $\begin{array}{l}\text { AGCGGTAAATCGTGGAGTGA } \\
\text { ACYTTACTGGTRCTGCACAT } \\
\text { CTACGGCACCACCAATGATA }\end{array}$ & Variable & 56 \\
\hline ORF513 & $\begin{array}{l}513 F \\
513 B \\
513 C\end{array}$ & $\begin{array}{l}\text { TGGAAGAGGGCGAAGACGAT } \\
\text { CGAATGCTCTGAGTCATCAT } \\
\text { TGCACTCTCTTTGTCACCAT }\end{array}$ & Variable & 52 \\
\hline IS26 & $\begin{array}{l}26 \mathrm{~F} \\
26 \mathrm{~B}\end{array}$ & $\begin{array}{l}\text { AGCGGTAAATCGTGGAGTGA } \\
\text { ACYTTACTGGTRCTGCACAT }\end{array}$ & Variable & 52 \\
\hline
\end{tabular}


PCR conditions were as above, using the annealing temperatures shown in Table 2. Primers with restriction enzyme digestion sites (CMF and CMB) and primers specific for the insertion sequences ISEcp1, IS903, ORF513 and IS26 are shown in Table 2.

To study the insertion sequence environment of our newly detected genetic subtypes of CTX-M ESBLs, the nested PCR amplification used the PCR product of the integrons as the template to detect insertion sequences with the $\mathrm{CMF}$ and $\mathrm{CMB}$ primer pair (Table 2). The CM primer pair with restriction enzyme digestion sites was self-designed. Long fragment PCR was used to amplify the entire sequence of CTX-M type ESBLs at the downstream side of ISEcP1B and the sequence was determined.

PCR products were sequenced by Shanghai Yingjun Biological and the results were analyzed with BLAST against GenBank to determine genotypes. PCR primers were synthesized by Shanghai Yingjun Biological and Takara Biotechnology (Dalian, China).

\section{Statistical analysis}

Data were expressed as numbers (\%). The analyses were carried out using the SPSS 15.0 software package (SPSS Institute, Chicago, IL, USA).

\section{RESULTS}

\section{Multi-drug resistance}

Drug resistance rates to 22 kinds of antibacterial drugs were tested. The total detection rate of multidrug-resistant Gram-negative bacilli was $26.49 \%$ ( 80 of 302 ). The drug to which the least number of multidrug-resistant isolates $(18.75 \%)$ were resistant was amikacin. The drug to which the most multidrug-resistant isolates $(97.50 \%)$ were resistant was ampicillin.

\section{Detection of $\beta$-lactamase by phenotype and genotype}

On the basis of phenotypic tests, majority of the 80 multidrugresistant isolates carried ESBLs (Table 3). Some strains carried ESBLs+AmpC or ESBLs+AmpC+MBL (Table 3). There was one strain for which the phenotype could not be determined.

Genotyping detected isolates carrying the TEM, SHV, CTX-M-G1, PSE, ACT-1, DHA-1, CMY and IMP-1 genotypes (Table 4). E. coli, K. pneumoniae, P. aeruginosa, Acinetobacter baumannii (A. baumannii), E. cloacae, Pseudomonas cepacia ( $P$. cepacia), Citrobacter freundii (C. freundii), Morganella morganii (M. morganii) and Flavobacterium meningosepticum (F. meningosepticum) carried 4-6 kinds of $\beta$-lactamase genes (Table 4 ), which is closely related to the multidrug resistance seen in clinical practice. Table 5 summarizes the combinations of $\beta$-lactamase genes that were carried by these strains.

\section{Detection of integrons}

To further understand the spread of multi-drug-resistance in our community, we looked for association of the $\beta$-lactamase genes with integrons that could serve as mechanisms of transmission for these genes. PCR using degenerate primers to amplify integrons from the 80 multi-drug resistant isolates detected class I integrons in 51 strains. Integrons were detected only in 30 strains when amplifying with the variable primers. Integrons were found in all genera of bacteria tested.

\section{Detection of insertion sequences}

Further, we used PCR amplification followed by sequencing of the PCR products to test for the presence of insertion sequences associated with the $\beta$-lactamase genes. The total detection rate of ISEcp $1 B$ was $16.25 \%$ (13 of 80 ). It was detected in $18.75 \%$ (3 of 16) of E. coli specimens, $25 \%$ (4 of 16) of K. pneumonia, 15.38\% (3 of 13) of P. aeruginosa, 25\% (2 of 8) of E. cloacae and 25\% (1 of 4 ) of C. freundii. Electrophoretic separation of the nested PCR products showed that the downstream side of the insertion sequence ISEcp $1 B$ connected with $b l a_{C T X-M-l i k e}$ genes, such as CTX-M-3 and CTX-M-9. The sequences of the PCR products of ISEcp $1 B$ were determined for eight stains. It was found that the $3^{\prime}$ end of most of the ISEcp $1 B$ sequences was connected with an inverted repeat sequence, which provides an action site for the translocase. The downstream side of that was connected with different CTX-M type ESBLs genes, which provided a recognition site for its horizontal transfer. In addition, two promoters, -35 and -10 , were present, which have an important regulatory role in the high-level expression and transmission of the downstream CTX-M-type ESBLs. The length of the start distance between the two promoters and the CTX-M-type ESBLs encoding gene varied, showing genetic polymorphism.

Sequencing results also revealed one new CTX-M ESBLs gene subtype, which is a composite transposon. It was composed of the genetic elements of ISEcp1B upstream of $b l a_{C T X-M-L i k e}$, and the

Table 3 The rate (\%) of phenotypic and genotypic detection of $\beta$-lactamase among 80 strains of multidrug-resistant Gram-negative bacilli

\begin{tabular}{|c|c|c|c|c|c|c|c|c|c|}
\hline \multirow[b]{2}{*}{ Pathogens } & \multirow[b]{2}{*}{$\mathrm{n}$} & \multicolumn{2}{|c|}{ With ESBLs production } & \multicolumn{2}{|c|}{ With ESBLs+AmpC production } & \multicolumn{2}{|c|}{ With AmpC production } & \multicolumn{2}{|c|}{ With $E S B L s+A m p C+M B L$ production } \\
\hline & & Phenotype & Genotype & Phenotype & Genotype & Phenotype & Genotype & Phenotype & Genotype \\
\hline ECO & 16 & $13(81.2)$ & $13(81.25)$ & $3(17.7)$ & $2(11.8)$ & $0(0.0)$ & $0(0.0)$ & $0(0.0)$ & $1(5.9)$ \\
\hline KPN & 16 & $12(75.0)$ & $12(75.0)$ & $3(18.8)$ & $3(18.8)$ & $0(0.0)$ & $0(0.0)$ & $2(18.8)$ & $2(18.8)$ \\
\hline PAE & 13 & $8(66.7)$ & $8(66.7)$ & $2(16.7)$ & $3(25.0)$ & $0(0.0)$ & $0(0.0)$ & $0(0.0)$ & $0(0.0)$ \\
\hline ABA & 10 & $10(100.0)$ & $9(90.0)$ & $0(0.0)$ & $0(0.0)$ & $0(0.0)$ & $0(0.0)$ & $0(0.0)$ & $0(0.0)$ \\
\hline ECL & 8 & $6(75.0)$ & $5(62.5)$ & $2(25.0)$ & $2(25.0)$ & $0(0.00)$ & $0(0.0)$ & $0(0.0)$ & $0(0.0)$ \\
\hline PCE & 4 & $2(50.0)$ & $3(75.0)$ & $2(50.0)$ & $1(25.0)$ & $0(0.0)$ & $0(0.0)$ & $0(0.0)$ & $0(0.0)$ \\
\hline CFR & 4 & $0(0.0)$ & $0(0.0)$ & $4(100.0)$ & $4(100.0)$ & $0(0.0)$ & $0(0.0)$ & $0(0.0)$ & $0(0.0)$ \\
\hline MMO & 2 & $0(0.0)$ & $0(0.0)$ & $2(100.0)$ & $2(100.0)$ & $0(0.0)$ & $0(0.0)$ & $0(0.0)$ & $0(0.0)$ \\
\hline FME & 3 & $0(0.0)$ & $0(0.0)$ & $3(100.0)$ & $3(100.0)$ & $0(0.0)$ & $0(0.0)$ & $0(0.0)$ & $0(0.0)$ \\
\hline FIN & 1 & $1(100.0)$ & $1(100.0)$ & $0(0.0)$ & $0(0.0)$ & $0(0.0)$ & $0(0.0)$ & $0(0.0)$ & $0(0.0)$ \\
\hline FOD & 1 & $1(100.0)$ & $1(100.0)$ & $0(0.0)$ & $0(0.0)$ & $0(0.0)$ & $0(0.0)$ & $0(0.0)$ & $0(0.0)$ \\
\hline EAE & 1 & $1(100.0)$ & $0(0.0)$ & $0(0.0)$ & $0(0.0)$ & $0(0.0)$ & $0(0.0)$ & $0(0.0)$ & $0(0.0)$ \\
\hline PMI & 1 & $1(100.0)$ & $1(100.0)$ & $0(0.0)$ & $0(0.0)$ & $0(0.0)$ & $0(0.0)$ & $0(0.0)$ & $0(0.0)$ \\
\hline Total & 80 & 56 & 53 & 21 & 20 & & & 2 & 3 \\
\hline
\end{tabular}

Abbreviations: ABA, Acinetobacter baumannii; CFR, Citrobacter freundii; EAE, Enterobacter aerogenes; ECO, Escherichia coli; ECL, Enterobacter cloacae; ESBL, extended-spectrum $\beta$-lactamase; FIN, Flavobacterium indologenes; FME, Flavobacterium meningosepticum; FOD, Flavobacterium odoratum; KPN, Klebsiella pneumoniae; MBL, metallo- $\beta$-lactamase; MMO, Morganella morganii; PAE, Pseudomonas aeruginosa; PCE, Pseudomonas cepacia; PMI, Proteus mirabilis. 
Table 4 The distribution of genotypic detection of $\beta$-lactamase in multidrug-resistant Gram-negative bacilli by genera

\begin{tabular}{lrrrrrrrrr}
\hline Pathogens & $\mathrm{n}$ & TEM & SHV & CTX-G1 & PSE & ACT-1 & DHA-1 & CMY & IMP-1 \\
\hline ECO & 16 & 12 & 6 & 9 & - & - & 2 & - & - \\
KPN & 16 & 13 & 5 & 7 & 3 & - & 3 & - & - \\
PAE & 13 & 9 & 5 & 6 & 3 & - & 3 & - & 1 \\
ABA & 10 & 8 & 2 & 1 & - & - & - & - & - \\
ECL & 8 & 6 & 2 & 4 & - & 2 & - & - & - \\
PCE & 4 & 4 & 3 & 1 & - & - & 1 & - & - \\
CFR & 4 & 3 & 4 & 1 & - & - & 3 & 4 & - \\
MMO & 2 & 1 & 1 & 2 & - & 1 & 1 & - & - \\
FME & 3 & 3 & 1 & 1 & - & - & 3 & - & - \\
FIN & 1 & 1 & - & - & - & - & - & - & - \\
FOD & 1 & 1 & 1 & - & - & - & - & - & - \\
EAE & 1 & 1 & - & - & - & - & - & - & - \\
PMI & 1 & - & 1 & - & - & - & - & - & - \\
Total & 80 & 62 & 31 & 32 & 6 & 3 & 16 & 4 & 1
\end{tabular}

Abbreviations: ABA, Acinetobacter baumannii; CFR, Citrobacter freundii; EAE, Enterobacter aerogenes; ECO, Escherichia coli; ECL, Enterobacter cloacae; ESBL, extended-spectrum $\beta$ lactamase; FIN, Flavobacterium indologenes; FME, Flavobacterium meningosepticum; FOD, Flavobacterium odoratum; KPN, Klebsiella pneumoniae; MBL, metallo- $\beta$-lactamase; MMO,

Morganella morganii; PAE, Pseudomonas aeruginosa; PCE, Pseudomonas cepacia; PMI, Proteus mirabilis. conserved sequence of the insertion sequence IS903 downstream of bla $C T X-M-L i k e$.

The insertion sequence from isolate En 45 was found to share $99 \%$ homology with an ISEcp1B found in Paris, France (GenBank accession no. AJ416345). ${ }^{11}$ The sixth nucleotide was changed from $\mathrm{A} \rightarrow \mathrm{T}$, resulting in the change of Lys: AAA $\rightarrow$ Asn: AAT. The 1006th nucleotide changed from $\mathrm{G} \rightarrow \mathrm{A}$, and the termination code changed from UGA: TGA $\rightarrow$ TAA.

In addition, the IS26 insertion sequence was found in E. coli 3 and K. pneumonia 49 isolates. It contained the tetracycline gene found in E. coli 3, but no $\beta$-lactamase gene was located in either insertion sequence.

\section{DISCUSSION}

Our survey showed that, during the 2005-2006 time period, multidrug-resistance due to $\beta$-lactamases was prevalent in Guangzhou hospital bacterial isolates. Various types of $\beta$-lactamase genes were found spread throughout all the tested genera. We carried out additional testing for genetic elements (integrons and insertion sequences) that could mediate transfer of the $\beta$-lactamase genes. Integrons and insertion sequences were found distributed among the various bacterial isolates, strongly suggesting that horizontal

Table 5 Combinations of drug resistance genes detected among different pathogens

\begin{tabular}{|c|c|c|c|c|c|c|c|c|c|c|c|c|c|c|}
\hline Drug resistance genes & \multicolumn{13}{|c|}{ Pathogens } & Total \\
\hline TEM & 6 & 4 & - & 6 & 2 & 2 & - & - & - & 1 & - & 1 & - & 22 \\
\hline SHV & - & 1 & - & 2 & 1 & - & - & - & - & - & - & - & 1 & 5 \\
\hline PSE & - & 1 & - & - & - & - & - & - & - & - & - & - & - & 1 \\
\hline $\mathrm{TEM}+\mathrm{CMY}$ & - & - & - & - & - & - & 1 & - & - & - & - & - & - & 1 \\
\hline TEM+SHV & 1 & 2 & 1 & - & - & - & - & - & - & - & 1 & - & - & 5 \\
\hline TEM+CTX-G1 & $\overline{2}$ & 2 & 1 & - & 1 & - & - & 1 & - & - & - & - & - & 7 \\
\hline TEM+DHA-1 & - & - & 2 & - & - & 1 & - & - & 1 & - & - & - & - & 3 \\
\hline TEM+ACT-1 & - & - & - & 1 & 2 & - & - & - & - & - & - & - & - & 3 \\
\hline $\mathrm{SHV}+\mathrm{CMY}$ & - & - & - & - & - & - & 1 & - & - & - & - & - & - & 1 \\
\hline \multicolumn{15}{|l|}{3 Genes } \\
\hline $\mathrm{SHV}+\mathrm{CTX}-\mathrm{G} 1+\mathrm{DHA}-1$ & 2 & - & - & - & - & - & - & - & - & - & - & - & - & 2 \\
\hline TEM+CTX-G1+DHA-1 & - & 2 & - & - & - & - & - & - & 1 & - & - & - & - & 3 \\
\hline SHV+CTX-G1+PSE & - & 1 & - & - & - & - & - & - & - & - & - & - & - & 1 \\
\hline TEM+ PSE+IMP1 & - & - & 1 & - & - & - & - & - & - & - & - & - & - & 1 \\
\hline $\mathrm{TEM}+\mathrm{SHV}+\mathrm{CTX}-\mathrm{G} 1$ & 1 & 1 & 2 & - & 1 & 1 & - & - & - & - & - & - & - & 6 \\
\hline $\mathrm{SHV}+\mathrm{CTX}-\mathrm{G} 1+\mathrm{CMY}$ & - & - & - & - & - & - & 1 & - & - & - & - & - & - & 1 \\
\hline TEM+ DHA-1+ CMY & - & - & - & - & - & - & 1 & - & - & - & - & - & - & 1 \\
\hline \multicolumn{15}{|l|}{4 Genes } \\
\hline TEM+SHV+DHA-1+CMY & - & - & - & - & - & - & - & - & 1 & - & - & - & - & 1 \\
\hline
\end{tabular}

Abbreviations: ABA, Acinetobacter baumannii; CFR, Citrobacter freundii; EAE, Enterobacter aerogenes; ECL, Enterobacter cloacae; ECO, Escherichia coli; FIN, Flavobacterium indologenes; FME, Flavobacterium meningosepticum; FOD, Flavobacterium odoratum; KPN, Klebsiella pneumoniae; MMO, Morganella morganii; PAE, Pseudomonas aeruginosa; PCE, Pseudomonas cepacia; PMI, Proteus mirabilis. 
transfer is responsible for the wide distribution of the $\beta$-lactamase genes that we observed. In our screen for integrons, we detected more varieties of amplicons than was previously reported for the Guangzhou region. ${ }^{12,13}$

The highly efficient integration and expression of integrons can enable the transmission of a drug resistance gene among bacteria in the same or different genera. ${ }^{3}$ The movement of transposons, such as ISEcp 1B, may promote resistance to gene evolution. ${ }^{14,15}$ The identification of a new CTX-M ESBLs gene subtype, the ISEcp1B/IS903 hybrid, which is a composite of known insertion sequences is evidence that evolution is occurring during these horizontal transfers. Similar composite transposons consisting of ISEcp1B and IS903 flanking the CTX-M-1, CTX-M-9, CTX-M-15, CTX-M-17 and CTX-M-54 genes, were reported in Europe, the Americas and Asia., ${ }^{70,14}$

We previously participated in a drug resistance monitoring group organized by the 13 major hospitals in the Guangzhou region from July 2001 to August 2003 and obtained 3500 strains of non-repetitive continuous isolates of Gram-negative bacilli. Of these 31\% (1084 of 3500) were positive for the ESBL phenotype. In our study we found that a similar percentage (26.49\%) of samples were multidrug-resistant.

A review of worldwide multi-drug resistance showed that there is a haphazard distribution of both nosocomial bacterial species and of the phenotype of resistance against antibiotics. ${ }^{16}$ In general ESBL phenotypes were rare in the 1980s and became prevalent in the 1990s. ${ }^{16}$ Regarding ESBL genotypes, the CTX-M family of ESBLs has become prevalent in the 2000s. ${ }^{17}$ Our data also shows a variety of phenotypic and genotypic $\beta$-lactamase genes and gene transmission elements. We found CTX-M family members and evidence of a new CTX-M variety. Thus, our results are similar to the prevailing trends of antibiotic resistance. Our genotypic data showing which genes exist together in clinical isolates (Table 5) may be valuable when developing antibiotic strategies. The presence of mobile genetic elements harboring multiple resistance genes enhances the survival of the mobile elements by providing multiple opportunities for selective pressure as the bacteria encounter various antibiotics. ${ }^{18}$ Rotation of the antibiotics used in a hospital has been proposed as a strategy to combat the spread of multidrug resistance, ${ }^{18}$ but successful implementation would require knowing which resistances are genetically linked so that the linked genes are not inadvertently maintained.

Our smaller sample size limits the accuracy of our statistics. Another limitation was that both the genotypic and phenotypic tests did not detect $\beta$-lactamase in some samples. This may have been because of the primer designs or PCR reaction conditions being unable to detect the genotype carried by certain strains. Our PCRbased tests to detect integrons also lacked sensitivity. To compensate for this we used both variable and degenerate primers, allowing us to detect more insertion sequences. The degenerate primers can potentially detect Class I, II and II integrons, whereas the variable primers only detect Class I integrons. This may explain the difference in detection rate.
Among the commonly used antibacterial drugs in clinical practice, bacteria had the lowest total drug resistance rate to amikacin (16 of 80 ), followed by imipenem (17 of 80 ) and piperacillin/tazobactam ( 24 of 80 ). These medicines should be the best choice to combat the extant multidrug-resistant strains. However, these antimicrobials should be judiciously rationed to prevent the development of further resistance. Continued clinical monitoring for multidrug-resistant bacteria is warranted.

\section{CONFLICT OF INTEREST}

The authors declare no conflict of interest.

1 Ambler, R. P. The structure of beta-lactamases. Philos. Trans. R. Soc. Lond. B. Biol. Sci. 289, 321-331 (1980).

2 Bush, K., Jacoby, G. A. \& Medeiros, A. A. A functional classification scheme for beta-lactamases and its correlation with molecular structure. Antimicrob. Agents. Chemother. 39, 1211-1233 (1995).

3 Rowe-Magnus, D. A. \& Mazel, D. The role of integrons in antibiotic resistance gene capture. Int. J. Med. Microbiol. 292, 115-125 (2002).

4 Fluit, A. C. \& Schmitz, F. J. Resistance integrons and super-integrons. Clin. Microbiol. Infect. 10, 272-288 (2004).

5 Hall, R. M. \& Collis, C. M. Antibiotic resistance in Gram-negative bacteria: the role of gene cassettes and integrons. Drug. Resist. Updat. 1, 109-119 (1998).

6 Clinical and Laboratory Standards Institute. Performance Standards for Antimicrobial Susceptibility Testing; 17th informational supplement. M100-S17 (Clinical and Laboratory Standards Institute, Wayne, PA, 2007).

7 Wu, W., Chen, M. \& Wang, H. Detection of derepressed continuous high-yielding AmpC enzyme and ultra broad spectrum $\beta$-lactamase in Enterobacter cloacae. Chin. J. Clin. Pharmacol. 17, 104-109 (2001).

8 Zhang, Y. et al. Drug resistance and genotype of $\beta$-lactamase in bacteria producing AmpC enzyme mediated by plasmids and ESBLs. Zhonghua. Microbiol. Immunol. J. 24, 577-582 (2004).

9 Xiao, Q., Su, D., Jiang, J. \& Zhong, N. Genotyping study of CTX-M-type and OXA-type broad-spectrum $\beta$-lactamase in Gram-negative bacilli in Guangzhou region. Chin. J. Hosp. Infect. 15, 1321-1326 (2005).

$10 \mathrm{Han}, \mathrm{L}$. et al. Two new integrons detected in one strain of Pseudomonas aeruginosa. Zhonghua. Microbiol. Immunol. J. 25, 485-489 (2005).

11 Eckert, C. et al. Dissemination of CTX-M-type beta-lactamases among clinical isolates of Enterobacteriaceae in Paris, France. Antimicrob. Agents Chemother. 48, 1249-1255 (2004).

12 Jiang, Y., Han, L., Jintang, J., Sun, J. \& Ni, Y. Distribution of class I integron in Gram-negative bacilli and its relationship with the drug resistance. Chin. J. Lab. Med. 28, 280-282 (2005).

$13 \mathrm{Xiao}, \mathrm{Z}$. et al. The screening of clinical bacterial resistance integron gene using multi-PCR. J. Jinan. Univ. 26, 480-485 (2005).

14 Lartigue, M. F., Poirel, L., Aubert, D. \& Nordmann, P. In vitro analysis of ISEcp1 B-mediated mobilization of naturally occurring beta-lactamase gene blaCTX-M of Kluyvera ascorbata. Antimicrob. Agents Chemother. 50, 1282-1286 (2006).

15 Cao, V., Lambert, T. \& Courvalin, P. ColE1-like plasmid pIP843 of Klebsiella pneumoniae encoding extended-spectrum beta-lactamase CTX-M-17. Antimicrob. Agents Chemother. 46, 1212-1217 (2002).

16 Winokur, P. L., Canton, R., Casellas, J. M. \& Legakis, N. Variations in the prevalence of strains expressing an extended-spectrum beta-lactamase phenotype and characterization of isolates from Europe, the Americas, and the Western Pacific region. Clin. Infect. Dis. 32 (Suppl 2), S94-S103 (2001).

17 Pitout, J. D. \& Laupland, K. B. Extended-spectrum beta-lactamase-producing Enterobacteriaceae: an emerging public-health concern. Lancet. Infect. Dis. 8, 159-166 (2008).

18 Canton, R., Coque, T. M. \& Baquero, F. Multi-resistant Gram-negative bacilli: from epidemics to endemics. Curr. Opin. Infect. Dis. 16, 315-325 (2003). 